\title{
Liver expression quantitative trait loci: a foundation for pharmacogenomic research
}

\author{
Dylan M. Glubb ${ }^{1}$, Neepa Dholakia ${ }^{2}$ and Federico Innocenti ${ }^{1,3 *}$ \\ 1 Institute for Pharmacogenomics and Individualized Therapy, University of North Carolina at Chapel Hill, Chapel Hill, NC, USA \\ 2 Eshelman School of Pharmacy, University of North Carolina at Chapel Hill, Chapel Hill, NC, USA \\ ${ }^{3}$ Lineberger Comprehensive Cancer Center, University of North Carolina at Chapel Hill, Chapel Hill, NC, USA
}

\section{Edited by:}

Ulrich M. Zanger, Dr. Margarete

Fischer-Bosch-Institute of Clinical

Pharmacology, Germany

Reviewed by:

Todd Skaar, Indiana University

Medical Center, USA

Rubin Lubomirov, University of

Lausanne, Switzerland

Vishal Lamba, University of

Minnesota, USA

\section{*Correspondence:}

Federico Innocenti, Eshelman

School of Pharmacy, Institute for

Pharmacogenomics and

Individualized Therapy, University of

North Carolina at Chapel Hill,

120 Mason Farm Road, Chapel Hill,

NC 27599, USA.

e-mail:innocent@email.unc.edu
Expression quantitative trait loci (eQTL) analysis can provide insights into the genetic regulation of gene expression at a genomic level and this information is proving extremely useful in many different areas of research. As a consequence of the role of the liver in drug metabolism and disposition, the study of eQTLs in primary human liver tissue could provide a foundation for pharmacogenomics. Thus far, four genome-wide eQTL studies have been performed using human livers. Many liver eQTLs have been found to be reproducible and a proportion of these may be specific to the liver. Already these data have been used to interpret and inform clinic genome-wide association studies, providing potential mechanistic evidence for clinical associations and identifying genes which may impact clinical phenotypes. However, the utility of liver eQTL data has not yet been fully explored or realized in pharmacogenomics. As further liver eQTL research is undertaken, the genetic regulation of gene expression will become much better characterized and this knowledge will create a rational basis for the prospective pharmacogenomic study of many drugs.

Keywords: liver, expression quantitative trait loci, ADME genes, GWAS, clinical pharmacogenomics

\section{INTRODUCTION}

As research has progressed, so has our understanding of how the genome plays a role in drug disposition and response via genes related to drug targets or absorption, distribution, metabolism, and excretion (ADME) (Goldstein et al., 2007). Indeed, germline human genetic variation is well known to affect drug disposition and clinical responses to pharmacotherapy. The Pharmacogenomics Knowledgebase (PharmGKB; www. pharmgkb.org) curates pharmacogenetic findings from the literature and, as of May 2012, lists 411 associations between germline genetic variants and drug-related phenotypes. Only $20 \%$ of these associations can be explained by genetic variation which leads to distinct protein differences through altered amino acid sequences. However, genetic variation can impact ADME gene expression in many different ways, at either mRNA or protein level (Glubb and Innocenti, 2011). Moreover, transcript levels provide clearly defined and measurable traits which may act as intermediates for drug-related phenotypes.

Genetic variants which associate with gene expression are known as expression quantitative trait loci (eQTLs) and can be further characterized as cis or trans. cis-eQTLs are located near the expressed gene, usually defined by an arbitrary distance (Gilad et al., 2008), whilst trans-eQTLs are located further away, sometimes on a different chromosome to the expressed gene. eQTLs can refer to any type of genetic variation but most often indicate that a single nucleotide polymorphism (SNP) genotype is associated with the transcript level of a gene. The interrogation of SNP genotypes and transcript levels have generally been performed using oligonucleotide microarray platforms, enabling the identification of eQTLs through genome-wide analyses (Franke and Jansen, 2009). This linking of gene variation and genetic expression may clarify the pharmacogenetic significance of the gene of interest (Li and Deng, 2010) and, thus, eQTLs have significant potential utility for the study of pharmacogenomics.

Lymphoblastoid cell lines (LCLs) have been the predominant model for eQTL analyses in humans (Skelly et al., 2009). These cell lines are derived from B-lymphocytes transformed by the Epstein-Barr virus (EBV). Although this transformation provides an immortalized cell line for study, which can be directly examined in pharmacogenomic studies (Huang et al., 2007a,b; 2008), there are limitations to their use as non-genetic factors can affect gene expression (Choy et al., 2008). These factors include the life history of the human donor, the B-lymphocyte subtype selected, the EBV titers used for transformation, the culture conditions in which the LCLs are grown and the intrinsic characteristics of the cell line. Nevertheless, several studies have translated LCL eQTL findings in clinical pharmacogenomic studies [reviewed in (Wheeler and Dolan, 2012)].

In the context of clinical pharmacogenomics, the liver may be the most relevant tissue in which to perform eQTL analyses. The liver is the predominant organ in drug elimination, with $75 \%$ of the 200 most widely prescribed drugs being eliminated from the body through liver metabolism or biliary excretion (Wienkers and Heath, 2005). Furthermore, a significant number of ADME genes are strongly expressed in the liver. Schroder et al. defined a list of 682 ADME genes from various pharmacogenomic resources 
(Schroder et al., 2011) and the expression of 61 of these genes have been predicted to be liver-specific according to a study of gene expression in human tissues (Yu et al., 2006). Therefore, eQTLs of these genes may not be detected in other tissues. In addition, eQTLs may have effects at the molecular level that are dependent upon the cell or organ in question. In this review, the first to our knowledge to focus specifically on liver eQTLs, we aim to show that the liver eQTL knowledge could provide a foundation to clinical pharmacogenomic research.

\section{LIVER eOTL STUDIES}

We are presently in the early stages of liver eQTL research and only four genome-wide eQTL studies have been reported which describe the analysis of primary human liver tissue (Table 1). However, several thousand eQTLs have been identified after correction for multiple testing and many of the eQTL SNPs (eSNPs) associate with the expression of ADME genes.

\section{LIVER EOTLS IN PHARMACOGENETIC STUDIES AND CLINICAL GENOME-WIDE ASSOCIATION STUDIES (GWAS)}

ADME genes with liver eQTLs, which pass multiple correction thresholds in one of the four genome-wide studies, include COMT, CYP2D6, CYP3A4, CYP3A5, MTHFR, UGT1A1, and VKORC1 (Table 2). These genes belong to a set of 'very important pharmacogenes' which have been identified by the PharmGKB resource (http://www.pharmgkb.org/search/browseVip.action? browseKey=vipGenes). Furthermore, several of these eSNPs are markers of pharmacogenetic associations (Table 2). These findings show that liver eQTLs may help explain the associations of SNPs with clinical phenotypes and also provide a rationale for using liver eQTL data from relevant genes to select candidate SNPs for pharmacogenomic study. For example, the three eSNPs identified in the CYP2D6 gene (Table 2) could conceivably be used to test associations with pertinent clinical phenotypes of any of the 46 drugs that, according to PharmGKB curated information (http://www.pharmgkb.org/gene/PA128\#tabview=tab6\& subtab $=21$ ), are metabolized by the CYP2D6 enzyme.

Liver eQTL data can also be used to inform GWAS. GWAS have identified many SNPs which are associated with clinical traits in humans. Typically there is no mechanistic explanation for the association but eQTLs could help fill this gap in the knowledge. Amongst the liver eQTL studies, Greenawalt et al. identified 122 eSNPs which were significantly associated, after multiple test correction, with a clinical phenotype in a GWAS (Greenawalt et al., 2011) and we found 32 eSNPs that were significant GWAS SNPs or markers for a GWAS SNP through strong linkage disequilibrium (LD) $\left(r^{2}>0.8\right)$ (Innocenti et al., 2011). In fact, there is a significant enrichment of eSNPs among SNPs associated with clinical phenotypes in GWAS (Nicolae et al., 2010). However, a critical piece of information which is lacking from these reports is the directionality of the eQTL association (i.e., which allele associates with higher/lower transcript levels) and, thus, hypotheses about the effect of a specific allele cannot be generated.

Another use for eQTLs is in determining which gene a SNP, associated with a clinical phenotype, may mediate its effect through as there is often uncertainty about GWAS SNPs located in intergenic regions. Schadt et al. provide examples of intergenic eSNPs that were found to associate with type 1 diabetes, coronary artery disease and LDL cholesterol levels in GWAS (Schadt et al., 2008). By identifying the gene whose expression the GWAS SNP associates with, evidence is provided to suggest that the gene may be a susceptibility factor for the disease.

\section{LIVER EOTL REPRODUCIBILITY}

There are many factors related to the liver eQTL studies which could act as confounders and affect eQTL reproducibility. Firstly,

Table 1 | Summary of human liver eQTL studies.

\begin{tabular}{|c|c|c|c|c|}
\hline Category & Schadt et al. & Innocenti et al. & Schroder et al. & Greenawalt et al. \\
\hline $\begin{array}{l}\text { Samples in final } \\
\text { analysis ( } n)\end{array}$ & 384 & 206 & 149 & 651 \\
\hline Race & All Caucasian & $\begin{array}{l}183 \text { European American } \\
23 \text { African American }\end{array}$ & All Caucasian & $\begin{array}{l}86 \% \text { of overall cohort } \\
\text { Caucasian }\end{array}$ \\
\hline Nature of liver tissue & $\begin{array}{l}\text { Postmortem tissue and } \\
\text { resections from donor livers }\end{array}$ & $\begin{array}{l}\text { Postmortem tissue and } \\
\text { resections from donor livers }\end{array}$ & $\begin{array}{l}\text { Normal tissue resected } \\
\text { during surgery for liver } \\
\text { cancer }\end{array}$ & $\begin{array}{l}\text { Tissue from morbidly obese } \\
\text { patients undergoing gastric } \\
\text { bypass }\end{array}$ \\
\hline Covariates & Age, sex and medical center & $\begin{array}{l}\text { Age, sex, genetic ancestry, } \\
\text { effects of array hybridization } \\
\text { and oligonucleotide probes, } \\
\text { and surrogate variables }\end{array}$ & $\begin{array}{l}\text { Age, sex, smoking, alcohol } \\
\text { consumption, diagnosis, } \\
\text { C-reactive protein, } \\
\text { cholestatic liver disease and } \\
\text { presurgical medication }\end{array}$ & Age, sex and medical center \\
\hline $\begin{array}{l}\text { Genotyping platform } \\
\text { (GEO dataset) }\end{array}$ & $\begin{array}{l}\text { Affymetrix } 500 \text { k Illumina } \\
650 Y \text { (GSE\# n/a) }\end{array}$ & $\begin{array}{l}\text { Illumina } 610 \text { Quad } \\
\text { (GSE26105) }\end{array}$ & $\begin{array}{l}\text { Illumina HumanHap300 } \\
\text { (GSE39036) }\end{array}$ & $\begin{array}{l}\text { Illumina 650Y } \\
\text { (GSE\# n/a) }\end{array}$ \\
\hline $\begin{array}{l}\text { Expression platform } \\
\text { (GEO dataset) }\end{array}$ & $\begin{array}{l}\text { Agilent Custom 44k } \\
\text { (GSE9588) }\end{array}$ & $\begin{array}{l}\text { Agilent } 4 \times 44 k \\
\text { (GSE25935) }\end{array}$ & $\begin{array}{l}\text { Illumina HumanWG-6 v2.0 } \\
\text { (GSE32504) }\end{array}$ & $\begin{array}{l}\text { Agilent Custom 44k } \\
\text { (GSE24293) }\end{array}$ \\
\hline cis-eQTLs (n) & $\begin{array}{l}1350 \text { (Bonferroni adjusted) } \\
3210 \text { (FDR<10\%) }\end{array}$ & $1787(\mathrm{BF}>5)$ & 1179 (Bonferroni adjustment) & 7902 (FDR<10\%) \\
\hline trans-eOTLs (n) & $\begin{array}{l}242 \text { (Bonferroni adjustment) } \\
491 \text { (FDR }<10 \% \text { ) }\end{array}$ & $353(B F>5)$ & 47 (Bonferroni adjustment) & $785($ FDR $<10 \%)$ \\
\hline
\end{tabular}


Table 2 | Liver eQTLs of PharmGKB 'very important pharmacogenes'a .

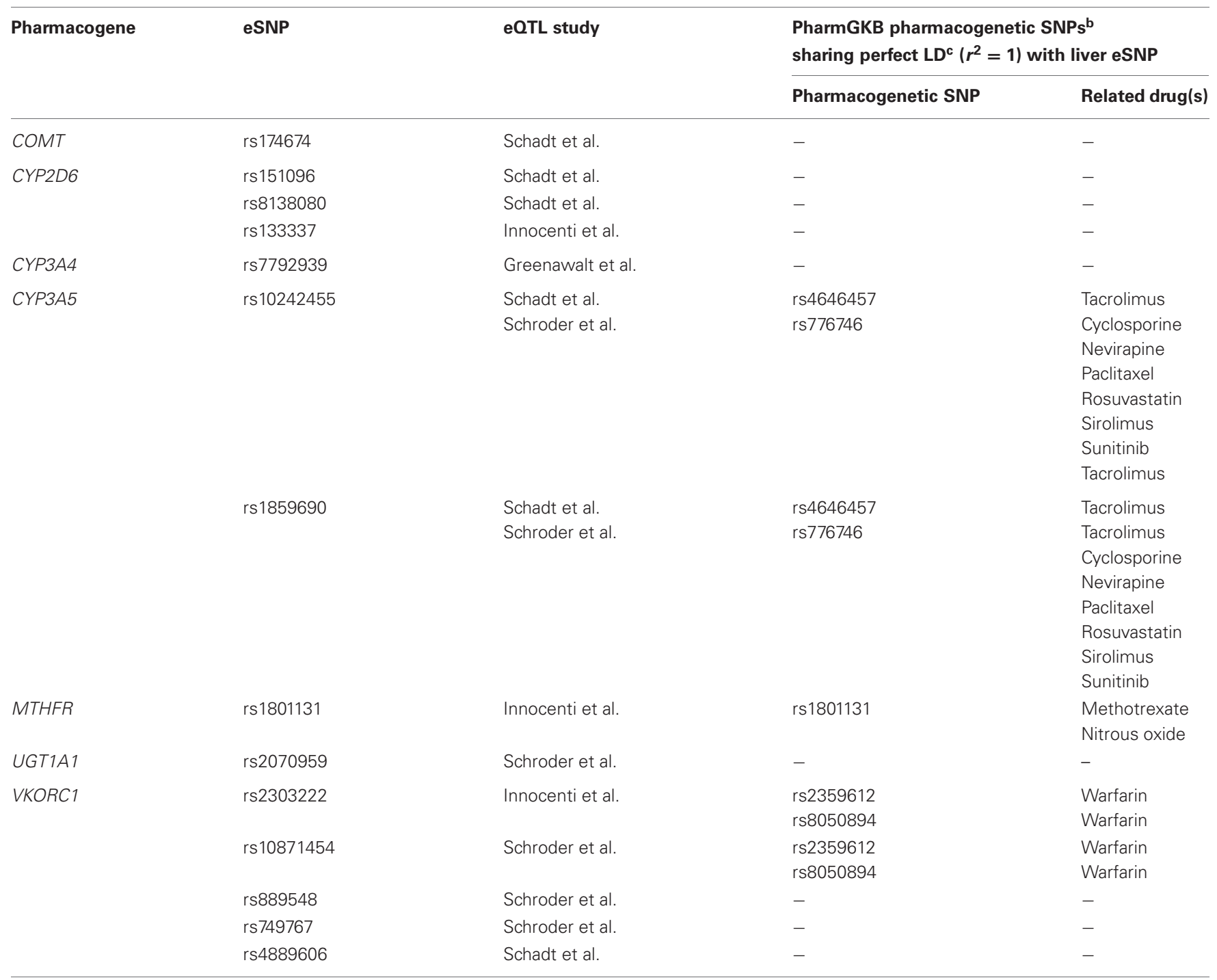

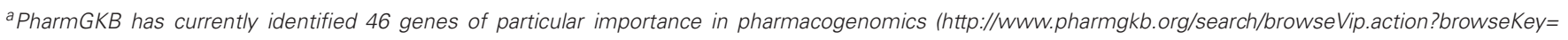
vipGenes).

${ }^{b}$ From the PharmGKB curated list of level 1-3 SNP-drug clinical annotations (http://www.pharmgkb.org/search/clinicalAnnotationList.action?evidenceStrength=1).

cLD determined from 1000 Genomes Pilot 1 CEU data using SNAP browser (http://www.broadinstitute.org/mpg/snap/ldsearch.php).

the liver eQTL studies use a variety of tissue sources from individuals with different ethnic backgrounds (Table 1). Tissue sources include resections of normal tissue from patients suffering from liver cancer (Schroder et al., 2011) or morbid obesity (Greenawalt et al., 2011). Resections of tissue from healthy and deceased liver donors, who may have undergone multiple pharmacotherapeutic treatments immediately prior to death, have also been used (Schadt et al., 2008; Innocenti et al., 2011). Secondly, different genotyping and gene expression platforms, and analysis methods were used to generate eQTLs (Table 1). Furthermore, these studies control for different covariates and have variable powering due to the number of samples, genotypes and transcripts interrogated (Table 1). Despite these issues, there is evidence that a substantial proportion of the cis-eQTL findings are reproducible. In our liver eQTL study, we attempted to replicate eQTL findings in two additional datasets, one of which was a subset of samples from the Schadt et al. study (Innocenti et al., 2011). 67\% of the significant cis-eQTLs (Bayes factor $(\mathrm{BF})>5$ ) replicated in at least one of the additional datasets ( $p<0.05$ and concordant effect direction), but this replication rate dropped to $6 \%$ for trans-eQTLs $(\mathrm{BF}>5$ ) (Innocenti et al., 2011). Greenawalt et al. examined the reproducibility of their liver cis-eQTLs (FDR $<10 \%)$ in the Schadt et al. study, which had used the same microarray gene expression platform, and found a $66 \%$ overlap (Greenawalt et al., 2011). Using Bonferroni adjusted pvalues, a much more stringent analysis, a comparison between the Schadt et al. and Schroder et al. studies showed that only $16 \%$ of cis-eQTLs replicated and no replication of trans-eQTLs 
(Schroder et al., 2011). Neither of the latter two studies state whether a concordant effect direction was a criterion for replication but it appears that liver cis-eQTLs are comparatively reproducible whilst trans-eQTLs are not. This observation may be partly explained by statistical power. Compared with cis-eQTLs, trans-eQTLs have a greater multiple test correction burden and, thus, a trans-eQTL is less likely to achieve statistical significance in two independent studies.

A salient finding in our study was that the eSNP with the highest BF was frequently located immediately upstream or downstream of the transcription start site (TSS) of the eQTL gene. Our group discovered that $75 \%$ of cis-eQTLs within $100 \mathrm{~kb}$ of the TSS were found to replicate, compared to $61 \%$ of those located more than $100 \mathrm{~kb}$ from the TSS (Innocenti et al., 2011). Furthermore, Schadt et al. noted in their study that $70 \%$ of the cis-eQTLs were found within $100 \mathrm{~kb}$ of the TSS (Schadt et al., 2008). Therefore, in general, it appears that reproducible liver cis-eQTL SNPs will be found close to gene TSSs. This suggests that these eSNPs may directly impact gene transcription by altering gene regulatory regions.

\section{LIVER eOTL TISSUE SPECIFICITY}

To examine tissue specificity of liver eQTLs, Schadt et al. compared their findings with eQTL analyses of human blood and adipose tissue (Emilsson et al., 2008). Approximately 30\% of the cis-eQTLs identified in these tissues were also detected among the liver cis-eQTLs (FDR $<10 \%)$ from their study (Schadt et al., 2008). Greenawalt et al., examined replication of their liver ciseQTLs in adipose tissue from the same cohort of individuals used for liver eQTL analysis and found 46-48\% of the liver ciseQTLs replicated at $p<0.05$ (Greenawalt et al., 2011). These observations suggest that some fraction of the genetic control of gene expression is specific to the liver. Indeed, Greenawalt et al. found that liver eQTLs, but not eQTLs identified from adipose tissue, were enriched for the KEGG pathway drug metabolismcytochrome P450 genes. Furthermore, of the 11 ADME genes which had reproducible eQTLs between the Schroder et al. and Schadt et al. studies (Schadt et al., 2008; Schroder et al., 2011), CYP3A5, DHRS2 and SLC22A3 have been predicted to have liver-specific expression (Yu et al., 2006).

\section{CORRELATION OF LIVER eOTLS AND P450 ENZYME ACTIVITY}

Gene transcript levels are a useful intermediate phenotype to study but measurements of protein levels or enzyme activity may provide a more informative phenotype for pharmacogenomics. One of the advantages of the liver eQTL model is that subcellular fractions can be generated from the liver, allowing proteomic and biochemistry studies to be integrated with genomic and transcriptomic data from the same tissue. For example, Yang et al. examined the enzyme activity of eight P450 proteins using liver microsomal samples derived from the same cohort as Schadt et al. (2008), and found that the activities of the CYP450s were positively correlated with mRNA transcript levels after adjusting transcript and activity levels for age, sex and study site (Yang et al., 2010). Transcript levels explained 10-30\% of the variance in enzyme activity, thus, indicating that other biological factors, such as post-translational mechanisms, may also impact on activity levels. Fifty four enzyme activity QTLs (aQTLs) were identified for the eight P450 proteins: 24 SNPs were transaQTLs and 30 cis-aQTLs (FDR<10\%) (Yang et al., 2010). The cis-aQTL SNPs were all associated with CYP2D6 enzyme activity and 29 of these were found to be also cis-eSNPs of CYP2D6 $(\mathrm{FDR}<10 \%)$.

\section{WEB-BASED LIVER EOTL DATA RESOURCES}

The Gene Expression Omnibus (GEO) repository contains data from all the human liver eQTL studies (Table 1), although genotypes from the Schadt et al. and Greenawalt et al. studies are not available. The data cannot be easily interrogated and, thus, we plan to upload the liver eQTL findings of our study to the web-based SNP and CNV Association (SCAN) database (Gamazon et al., 2010). The results of the Schadt et al. study are already available through the Genotype-Tissue Expression (GTEx) eQTL browser (http://www.ncbi.nlm.nih. gov/gtex/GTEX2/gtex.cgi), and can be queried by gene or SNP and filtered by $p$-value or $r^{2}$. The GTEx browser provides further useful information by annotating corresponding eSNPs with significant findings from GWAS.

\section{FUTURE DIRECTIONS CNVs}

Single nucleotide base changes are the most common but not the only germline genetic variation present in the human genome. A study of genetic variation in eight individuals identified four million SNPs, $\sim 800,000$ insertion/deletions ( $>1-100$ bp) and 1695 structural $(>6 \mathrm{~kb})$ variants, also known as copy number variants (CNVs) (Kidd et al., 2008). Although CNVs are much fewer in number than SNPs, their potential to impact on gene expression is greater due to their size and ability to increase gene copy number or ablate the transcriptional unit. Indeed, SNPs which are in strong $\mathrm{LD}\left(r^{2}=0.8\right)$ with CNVs are more likely to be LCL eQTLs and associated with the expression of more than one gene (Gamazon et al., 2011). Moreover, the importance of CNVs in pharmacogenetics is already well known. CNVs with functional effects on enzyme activity of proteins (e.g., CYP2D6, GSTM1 and GSTT1) have been observed to associate with drug efficacy and toxicity (He et al., 2011). It is likely that these CNVs may be eQTLs and, thus, there is a great need for liver CNV eQTL analyses to be carried out in the future.

\section{NEXT-GENERATION SEQUENCING}

Previous liver eQTL studies have interrogated genetic variation and gene expression at the genome level. However, due to the limitations of the oligonucleotide genotyping and gene expression microarray platforms, this corresponds only to a fraction of human genetic variation and does not encompass all transcript splice variants or gene isoforms.

Genotype imputation can be performed using genotype data from studies like HapMap or 1000 Genomes Project to greatly increase the number of genotypes interrogated through oligonucleotide genotyping platforms. Indeed, in our liver eQTL study we imputed genotypes from HapMap to generate more than two million genotypes (Innocenti et al., 2011). While still relatively expensive, next-generation sequencing has advanced to the point 
where it is becoming feasible to perform whole genome sequencing of DNA and mRNA to comprehensively characterize genetic variation and the entire transcriptome in a relatively large number of samples.

Although there is high correlation between transcript levels quantitated by RNA-seq and microarrays (Pickrell et al., 2010), RNA-seq provides a greater resolution which leads to detection of more eQTLs (Montgomery et al., 2010). The RNA-seq (next generation sequencing of mRNA) approach has other advantages over oligonucleotide expression arrays e.g., no saturation of intensity signal for high abundance transcripts and the ability to quantify allele-specific expression (Majewski and Pastinen, 2011). Additionally, RNA-seq can identify splicing QTLs which may not affect overall gene expression but alter transcript isoform levels (Pickrell et al., 2010).

\section{IDENTIFICATION OF FUNCTIONAL eSNPS}

eQTL analyses are informative but they cannot definitively identify the causative SNPs that affect gene expression. Due to LD, many eSNPs can represent the same causative genetic variant. Various approaches can be used to identify functional genetic variants which generate the eQTL signal. Reporter gene assays are useful in determining whether eSNPs in promoter, $5^{\prime}$ UTR, $3^{\prime}$ UTR and intronic regions have functional effects on gene expression (Innocenti et al., 2011). However, this technique cannot be easily applied in a high-throughput fashion and the effects of SNPs in coding regions are generally not amenable to testing.

Bioinformatic analyses may help to narrow the number of putatively causative SNPs, by identifying eSNPs which are located in functional genomic elements such as those defined by the Encyclopedia of DNA Elements (ENCODE) Project (Myers et al., 2011). Indeed, a study of LCL eQTLs found that $40 \%$ of the eSNPs were located in open chromatin regions and sites of DNaseI hypersensitivity or histone modification. Furthermore, SNPs in DNaseI hypersensitivity sites were four-fold more likely to associate with transcript levels compared with other SNPs (Gaffney et al., 2012). This epigenetic, and other regulatory, information can be combined with eQTL data to create a model which ranks eSNPs on their potential to affect gene expression (Gaffney et al., 2012). This approach has not yet been applied to a study of liver eQTLs but could prove extremely useful in identifying pharmacogenetic variants.

\section{REFERENCES}

Becker, J., Wendland, J. R., Haenisch, B., Nothen, M. M., and Schumacher, J. (2012). A systematic eQTL study of cis-trans epistasis in 210 HapMap individuals. Eur. J. Hum. Genet. 20, 97-101.

Choy, E., Yelensky, R., Bonakdar, S., Plenge, R. M., Saxena, R., De Jager, P. L., Shaw, S. Y., Wolfish, C. S., Slavik, J. M., Cotsapas, C., Rivas, M., Dermitzakis, E. T., CahirMcFarland, E., Kieff, E., Hafler, D., Daly, M. J., and Altshuler, D. (2008). Genetic analysis of human traits in vitro: drug response and gene expression in lymphoblastoid cell lines. PLoS Genet. 4:e1000287. doi: 10.1371/journal.pgen. 1000287

Emilsson, V., Thorleifsson, G., Zhang, B., Leonardson, A. S., Zink, F., Zhu, J., Carlson, S., Helgason, A., Walters, G. B., Gunnarsdottir, S., Mouy, M., Steinthorsdottir, V., Eiriksdottir, G. H., Bjornsdottir, G., Reynisdottir, I., Gudbjartsson, D., Helgadottir, A., Jonasdottir, A., Styrkarsdottir, U., Gretarsdottir, S., Magnusson, K. P., Stefansson, H., Fossdal, R., Kristjansson, K., Gislason, H. G., Stefansson, T., Leifsson, B. G., Thorsteinsdottir, U., Lamb, J. R., Gulcher, J. R., Reitman, M. L., Kong, A., Schadt, E. E., and

\section{EPISTATIC EOTL INTERACTIONS}

eQTL analyses generally examine only the effects of a single genetic locus on gene expression. However, it is possible that interactions of cis- and trans-eQTLs contribute to the regulation of gene expression. Indeed, $15 \%$ of transcripts were found to be regulated by a cis-trans eQTL interaction in a study of HapMap LCLs and the vast majority of the eSNPs were not significantly associated with gene expression in a single locus analysis (Becker et al., 2012). Therefore, important information may be lost by not considering these eQTL interactions and this approach could be used to detect epistatic pharmacogenetic variants which may not otherwise be identified.

\section{LIVER PROTEIN QTLS}

The Yang et al. study (Yang et al., 2010) correlating liver eQTLs and P450 enzyme activity highlights the need for liver protein QTL analyses to characterize the genetic regulation of protein expression. High-throughput proteome-wide quantification of protein expression is much more problematic and less straightforward than that for transcriptome quantification. Liquid chromatography-tandem mass spectrometry (LC-MS/MS) techniques are providing a way forward but, currently, the best approach for sensitive and accurate protein quantitation may be to carry out targeted proteomic studies (Kline et al., 2009). For example, targeted LC-MS/MS analysis of several UGT1A isoforms has been successfully performed in human liver microsomes (Harbourt et al., 2012).

\section{CONCLUSION}

There is a strong rationale for using findings from human liver eQTL studies as a knowledge base to inform and guide clinical pharmacogenomic studies. Liver eQTL analyses provide insights into the genetic regulation of ADME genes which may not be obtained from other tissues and identifies eQTLs which are reproducible and unique to the liver. Moreover, liver eQTL data can help explain previously identified pharmacogenomic associations and provides candidates for prospective clinical study. In the future, the integration of epistatic, epigenetic, CNV, nextgeneration sequencing, bioinformatic, and protein analyses will generate a truly comprehensive map of the genetic regulation of gene expression in the liver which is certain to profoundly impact pharmacogenomics.

Stefansson, K. (2008). Genetics of gene expression and its effect on disease. Nature 452, 423-428.

Franke, L., and Jansen, R. C. (2009). eQTL analysis in humans. Methods Mol. Biol. 573, 311-328.

Gaffney, D. J., Veyrieras, J. B., Degner, J. F., Pique-Regi, R., Pai, A. A., Crawford, G. E., Stephens, M., Gilad, Y., and Pritchard, J. K. (2012). Dissecting the regulatory architecture of gene expression QTLs. Genome Biol. 13, R7.

Gamazon, E. R., Nicolae, D. L., and Cox, N. J. (2011). A study of CNVs as trait-associated polymorphisms and as expression quantitative trait loci. PLoS Genet. 7:e1001292. doi: 10.1371/journal.pgen.1001292

Gamazon, E. R., Zhang, W., Konkashbaev, A., Duan, S., Kistner, E. O., Nicolae, D. L., Dolan, M. E., and Cox, N. J. (2010). SCAN: SNP and copy number annotation. Bioinformatics 26, 259-262.

Gilad, Y., Rifkin, S. A., and Pritchard, J. K. (2008). Revealing the architecture of gene regulation: the promise of eQTL studies. Trends Genet. 24, 408-415.

Glubb, D. M., and Innocenti, F. (2011). Mechanisms of genetic regulation in gene expression: examples from drug metabolizing enzymes and 
transporters. Wiley Interdiscip. Rev. Syst. Biol. Med. 3, 299-313.

Goldstein, D. B., Need, A. C., Singh, R., and Sisodiya, S. M. (2007). Potential genetic causes of heterogeneity of treatment effects. Am. J. Med. 120, S21-S25.

Greenawalt, D. M., Dobrin, R., Chudin, E., Hatoum, I. J., Suver, C., Beaulaurier, J., Zhang, B., Castro, V., Zhu, J., Sieberts, S. K., Wang, S., Molony, C., Heymsfield, S. B., Kemp, D. M., Reitman, M. L., Lum, P. Y., Schadt, E. E., and Kaplan, L. M. (2011). A survey of the genetics of stomach, liver, and adipose gene expression from a morbidly obese cohort. Genome Res. 21, 1008-1016.

Harbourt, D. E., Fallon, J. K., Ito, S., Baba, T., Ritter, J. K., Glish, G. L., and Smith, P. C. (2012). Quantification of human uridine-diphosphate glucuronosyl transferase 1A isoforms in liver, intestine, and kidney using nanobore liquid chromatographytandem mass spectrometry. Anal. Chem. 84, 98-105.

He, Y., Hoskins, J. M., and McLeod, H. L. (2011). Copy number variants in pharmacogenetic genes. Trends Mol. Med. 17, 244-251.

Huang, R. S., Duan, S., Bleibel, W. K., Kistner, E. O., Zhang, W., Clark, T. A., Chen, T. X., Schweitzer, A. C., Blume, J. E., Cox, N. J., and Dolan, M. E. (2007a). A genome-wide approach to identify genetic variants that contribute to etoposide-induced cytotoxicity. Proc. Natl. Acad. Sci. U.S.A. 104, 9758-9763.

Huang, R. S., Duan, S., Shukla, S. J., Kistner, E. O., Clark, T. A., Chen, T. X., Schweitzer, A. C., Blume, J. E., and Dolan, M. E. (2007b). Identification of genetic variants contributing to cisplatin-induced cytotoxicity by use of a genomewide approach. Am. J. Hum. Genet. 81, 427-437.

Huang, R. S., Duan, S., Kistner, E. O., Bleibel, W. K., Delaney, S. M., Fackenthal, D. L., Das, S., and Dolan, M. E. (2008). Genetic variants contributing to daunorubicininduced cytotoxicity. Cancer Res. 68, 3161-3168.
Innocenti, F., Cooper, G. M., Stanaway, I. B., Gamazon, E. R., Smith, J. D., Mirkov, S., Ramirez, J., Liu, W., Lin, Y. S., Moloney, C., Aldred, S. F., Trinklein, N. D., Schuetz, E., Nickerson, D. A., Thummel, K. E., Rieder, M. J., Rettie, A. E., Ratain, M. J., Cox, N. J., and Brown, C. D. (2011). Identification, replication, and functional finemapping of expression quantitative trait loci in primary human liver tissue. PLoS Genet. 7:e1002078. doi: 10.1371/journal.pgen. 1002078

Kidd, J. M., Cooper, G. M., Donahue, W. F., Hayden, H. S., Sampas, N., Graves, T., Hansen, N., Teague, B., Alkan, C., Antonacci, F., Haugen, E., Zerr, T., Yamada, N. A., Tsang, P., Newman, T. L., Tuzun, E., Cheng, Z., Ebling, H. M., Tusneem, N., David, R., Gillett, W., Phelps, K. A., Weaver, M., Saranga, D., Brand, A., Tao, W., Gustafson, E., McKernan, K., Chen, L., Malig, M., Smith, J. D., Korn, J. M., McCarroll, S. A., Altshuler, D. A., Peiffer, D. A., Dorschner, M., Stamatoyannopoulos, J., Schwartz, D., Nickerson, D. A., Mullikin, J. C., Wilson, R. K., Bruhn, L., Olson, M. V., Kaul, R., Smith, D. R., and Eichler, E. E. (2008). Mapping and sequencing of structural variation from eight human genomes. Nature 453, 56-64.

Kline, K. G., Finney, G. L., and Wu, C. C. (2009). Quantitative strategies to fuel the merger of discovery and hypothesis-driven shotgun proteomics. Brief. Funct. Genomic. Proteomic. 8, 114-125.

Li, H., and Deng, H. (2010). Systems genetics, bioinformatics and eQTL mapping. Genetica 138, 915-924.

Majewski, J., and Pastinen, T. (2011). The study of eQTL variations by RNA-seq: from SNPs to phenotypes. Trends Genet. 27, 72-79.

Montgomery, S. B., Sammeth, M., Gutierrez-Arcelus, M., Lach, R. P., Ingle, C., Nisbett, J., Guigo, R., and Dermitzakis, E. T. (2010). Transcriptome genetics using second generation sequencing in a Caucasian population. Nature 464, 773-777.
Myers, R. M., Stamatoyannopoulos, J., Snyder, M., Dunham, I., Hardison, R. C., Bernstein, B. E., Gingeras, T. R., Kent, W. J., Birney, E., Wold, B., and Crawford, G. E. (2011). A user's guide to the encyclopedia of DNA elements (ENCODE). PLoS Biol. 9:e1001046. doi: 10.1371/journal.pbio. 1001046

Nicolae, D. L., Gamazon, E., Zhang, W. Duan, S., Dolan, M. E., and Cox, N. J. (2010). Trait-associated SNPs are more likely to be eQTLs: annotation to enhance discovery from GWAS. PLoS Genet. 6:e1000888. doi: 10.1371/journal.pgen.1000888

Pickrell, J. K., Marioni, J. C., Pai, A. A., Degner, J. F., Engelhardt, B. E., Nkadori, E., Veyrieras, J. B., Stephens, M., Gilad, Y., and Pritchard, J. K. (2010). Understanding mechanisms underlying human gene expression variation with RNA sequencing. Nature 464, 768-772.

Schadt, E. E., Molony, C., Chudin, E., Hao, K., Yang, X., Lum, P. Y., Kasarskis, A., Zhang, B., Wang, S., Suver, C., Zhu, J., Millstein, J., Sieberts, S., Lamb, J., Guhathakurta, D., Derry, J., Storey, J. D., AvilaCampillo, I., Kruger, M. J., Johnson, J. M., Rohl, C. A., Van Nas, A., Mehrabian, M., Drake, T. A., Lusis, A. J., Smith, R. C., Guengerich, F. P., Strom, S. C., Schuetz, E., Rushmore, T. H., and Ulrich, R. (2008). Mapping the genetic architecture of gene expression in human liver. PLoS Biol. 6:e107. doi: 10.1371/journal.pbio.0060107

Schroder, A., Klein, K., Winter, S. Schwab, M., Bonin, M., Zell, A., and Zanger, U. M. (2011). Genomics of ADME gene expression: mapping expression quantitative trait loci relevant for absorption, distribution, metabolism and excretion of drugs in human liver. Pharmacogenomics J. doi: 10.1038/tpj.2011.44. [Epub ahead of print].

Skelly, D. A., Ronald, J., and Akey, J. M. (2009). Inherited variation in gene expression. Annu. Rev. Genomics Hum. Genet. 10, 313-332.

Wheeler, H. E., and Dolan, M. E. (2012). Lymphoblastoid cell lines in pharmacogenomic discovery and clinical translation. Pharmacogenomics 13, 55-70.

Wienkers, L. C., and Heath, T. G. (2005). Predicting in vivo drug interactions from in vitro drug discovery data. Nat. Rev. Drug Discov. 4, 825-833.

Yang, X., Zhang, B., Molony, C., Chudin, E., Hao, K., Zhu, J., Gaedigk, A., Suver, C., Zhong, H., Leeder, J. S., Guengerich, F. P., Strom, S. C., Schuetz, E., Rushmore, T. H., Ulrich, R. G., Slatter, J. G., Schadt, E. E., Kasarskis, A., and Lum, P. Y. (2010). Systematic genetic and genomic analysis of cytochrome P450 enzyme activities in human liver. Genome Res. 20, 1020-1036.

Yu, X., Lin, J., Zack, D. J., and Qian, J. (2006). Computational analysis of tissue-specific combinatorial gene regulation: predicting interaction between transcription factors in human tissues. Nucleic Acids Res. 34, 4925-4936.

Conflict of Interest Statement: The authors declare that the research was conducted in the absence of any commercial or financial relationships that could be construed as a potential conflict of interest.

Received: 31 May 2012; paper pending published: 25 June 2012; accepted: 30 July 2012; published online: 14 August 2012.

Citation: Glubb DM, Dholakia $N$ and Innocenti $F$ (2012) Liver expression quantitative trait loci: a foundation for pharmacogenomic research. Front. Gene. 3:153. doi: 10.3389/fgene.2012.00153 This article was submitted to Frontiers in Pharmacogenetics and Pharmacogenomics, a specialty of Frontiers in Genetics.

Copyright (c) 2012 Glubb, Dholakia and Innocenti. This is an open-access article distributed under the terms of the Creative Commons Attribution License, which permits use, distribution and reproduction in other forums, provided the original authors and source are credited and subject to any copyright notices concerning any third-party graphics etc. 